\title{
Trajectory Optimization of Energy Efficient FSOC-UAV with Atmospheric and Geometric Loss
}

\author{
Ju-Hyung Lee ${ }^{\dagger}$, Ki-Hong Park ${ }^{\ddagger}$, Mohamed-Slim Alouini ${ }^{\ddagger}$ and Young-Chai Ko ${ }^{\dagger}$ \\ ${ }^{\dagger}$ Electrical and Computer Engineering, Korea University, Seoul, Korea \\ ${ }^{\ddagger}$ Computer, Electrical and Mathematical Science and Engineering (CEMSE) Division \\ King Abdullah University of Science and Technology (KAUST) Thuwal, Saudi Arabia \\ leejuhyung@korea.ac.kr, \{kihong.park,slim.alouini\}@kaust.edu.sa, koyc@korea.ac.kr
}

\begin{abstract}
This paper address the issues of optimizing unmanned aerial vehicle (UAV) using free-space optical communication (FSOC). In particular, we examine how the beam divergence and the atmospheric attenuation of FSOC channel affect the flight of energy efficient UAVs. Especially based on geometric loss and also the effect of rain, snow, and fog on FSOC, we showed the energy efficient trajectory of UAV.
\end{abstract}

\section{INTRODUCTION}

A unmanned aerial vehicle (UAV) can be used as a mobile terminal communicating with other terrestrial equipment or with other aerial vehicles. The UAV can freely move in three dimensions, allowing the vehicle to communicate more flexibly than fixed terminals. Accordingly, UAV-assisted communication can be useful not only for the public but also for particular situations, such as defense or disaster issues. In particular, UAV-assisted free space optical communication (FSOC) is currently being actively researched for the purpose of providing internet access in areas with the insufficient network connectivity. However, UAV has the limitation that it can not secure energy continuously. Therefore, it is necessary for the UAV to fly energy efficiently for an application that requires a certain amount of operating time. In [1] and [2], energy efficient UAV management scheme is proposed in the context of limited energy consumption.

Inspired by the above works on UAV communications, we solve energy efficient UAV route optimization problems that consider FSOC channel characteristics, unlike [1] and [2] for RF channel. Especially, taking into account the beam divergence and atmospheric environment, we find the energy efficient trajectories of UAV more suited to the actual operating condition.

\section{SySTEM MODEL}

It is important to note that we consider the energy efficiency in the discrete domain, to relax the infinite number of variables caused by continuous movements of UAV. With energyefficiency objective function, we optimize the UAV trajectory. Considering mobility of UAV, this system sets the problem in three-dimensional space. We assume that ground terminal located on $(0,0,0)$ and UAV flies horizontally with a constant altitude $H$. Based on the discrete time step, $t=n \delta_{t}, n=$ $0,1, \ldots, N-1$, the position vector is $\mathbf{q}[n]=[x[n], y[n]]^{T}$, and the distance between ground terminal and UAV is $d[n]=$ $\sqrt{H^{2}+\|\mathbf{q}[n]\|^{2}}$.

For energy consumption model, we use previously introduced upper bound of propulsion energy consumption model of fixed-wing UAV [1] as follow,

$$
E_{T}(\mathbf{q}[n])=\sum_{n=1}^{N}\left[c_{1}\|\mathbf{v}[n]\|^{3}+\frac{c_{2}}{\|\mathbf{v}[n]\|}\left(1+\frac{\|\mathbf{a}[n]\|^{2}}{g^{2}}\right)\right],
$$

which assumed with $\mathbf{a}^{T}[n] \mathbf{v}[n]=0$ and same initial, end velocity $\mathbf{v}_{0}=\mathbf{v}_{F}$. Note that energy for communication of UAV is much smaller than energy for movement of UAV and accordingly assumed to be negligible [2].

In this paper, the line-of-sight (LOS) link for FSOC is assumed and the multi-path is not considered. We note that the fading is not considered because we optimize the UAV trajectory prior to its operation. Accordingly, the channel gain is based on the path loss of FSOC depending on the position of the UAV.

An atmospheric loss in FSOC is mainly affected by beam divergence and weather conditions [3]. To describe the attenuation due to the fog, rain, and snow, the three coefficients are expressed as follows, $\tilde{\beta}_{\text {rain }}=1.076 R^{0.67}, \tilde{\beta}_{\text {snow }}=a S^{b}$, and $\tilde{\beta}_{\text {fog }}(\lambda)=\frac{3.91}{V}\left(\frac{\lambda}{550 \mathrm{~nm}}\right)^{-p}$, where $R, S$ are rainfall and snowfall rates, $V$ is visibility, $p$ is size distribution coefficient of scattering, $\lambda$ is wavelength and assumed $780 \mathrm{~nm}$ in this paper. Considering these three environmental factors, attenuation by weather condition is determined as follows, $\tilde{\beta}=\tilde{\beta}_{\text {rain }}+\tilde{\beta}_{\text {snow }}+\tilde{\beta}_{\text {fog }}(\lambda)[\mathrm{dB} / \mathrm{km}], \beta=\frac{\tilde{\beta}}{10^{4} \log _{10} e}\left[\mathrm{~m}^{-1}\right]$. For explaining the attenuation of propagation channel, we model channel $h=h_{a} h_{g} \cdot h_{a}=e^{-\beta d[n]}$ and $h_{g}=\frac{D_{R}^{2}}{\left(D_{T}+\theta_{\text {div }} d[n]\right)^{2}}$ describe the attenuations of the propagation channel due to weather and beam divergence, respectively, where $D_{T}$ and $D_{R}$ are aperture diameters of transmitter and receiver, and $\theta_{\mathrm{div}}$ is a divergence angle.

In this system, the data rate of FSOC between the UAV and ground terminal formulated as follow [4], $R_{T}(\mathbf{q}[n])=$ $\frac{B}{2} \sum_{n=1}^{N} \log \left(1+k_{1} e^{-k_{2} d[n]}\right)$ [nats], where $\Lambda$ and $\varepsilon$ are peak and average power, $\alpha=\frac{\varepsilon}{\Lambda}, \sigma$ is the noise variance, $\gamma=\frac{\varepsilon}{\sigma}$ is average signal-to-noise ratio (ASNR), $B$ is the bandwidth of FSOC communication, $k_{1}$ is $\frac{e^{2 \alpha \mu^{*}}}{2 \pi e}\left(\frac{1-e^{-\mu^{*}}}{\mu^{*}}\right)^{2} \frac{h_{g}^{2} \gamma^{2}}{\alpha^{2}}$ for $0<$ 
$\alpha<\frac{1}{2}, \frac{h_{g}^{2} \gamma^{2}}{2 \pi e \alpha^{2}}$ for $\frac{1}{2}<\alpha<1$ respectively. Note that $\alpha=$ $\frac{1}{\mu^{*}}-\frac{e^{-\mu^{*}}}{\left(1-e^{-\mu^{*}}\right)}$ and $k_{2}=2 \beta$ [4]. Considering the values related to the rate of FSOC and the energy of fixed-wing UAV, we define energy-efficiency as follows, $\operatorname{EE}(\mathbf{q}[n])=\frac{R_{T}(\mathbf{q}[n])}{E_{T}(\mathbf{q}[n])}$.

\section{ENERGY-EFFICIENCY MAXIMIZATION}

We optimize the energy-efficiency of UAV using FSOC, taking into account the actual operating environment. For this problem, we follow the solution to UAV energy-efficiency optimization problem using RF communication. However, the existing problem is expanded considering the inherent channel characteristics of FSOC.

Based on the Taylor approximation of velocities and acceleration formulas of UAV [1], we formulate the energy-efficiency maximization problem as follow,

$$
\begin{array}{cl}
\max _{\mathbf{q}[n], \mathbf{v}[n], \mathbf{a}[n]} & \operatorname{EE}(\mathbf{q}[n])) \\
\text { s.t } & \mathbf{v}[n+1]=\mathbf{v}[n]+\mathbf{a}[n] \delta_{t}, \\
& \mathbf{q}[n+1]=\mathbf{q}[n]+\mathbf{v}[n] \delta_{t}+\frac{1}{2} \mathbf{a}[n] \delta_{t}^{2}, \\
& \mathbf{q}[0]=\mathbf{q}_{\mathrm{I}}, \mathbf{q}[N+1]=\mathbf{q}_{\mathrm{F}}, \\
& \mathbf{v}[0]=\mathbf{v}_{\mathrm{I}}, \mathbf{v}[N+1]=\mathbf{v}_{\mathrm{F}}, \\
& \|\mathbf{v}[n]\| \leq V_{\max },\|\mathbf{a}[n]\| \leq a_{\max }, \\
& V_{\min } \leq\|\mathbf{v}[n]\|, \quad(n=1, \ldots, N) .
\end{array}
$$

Note that the numerator in (2) is non-concave and the numerator and the denominator in (2) and the constraint in (8) are non-convex. We approximate the non-concave part to concave using high-SNR approximation, and also the nonconvex part to a convex problem using a slack variable, and the first Taylor approximation as in [1]. Considering the upper bound of (1) and the rate model for FSOC, the energyefficiency problem (2) is reformulated as

$$
\begin{aligned}
\max _{\substack{\mathbf{q}[n], \mathbf{v}[n] \\
\mathbf{a}[n], \tau[n]}} & \frac{B}{2} \sum_{n=1}^{N}\left(\log \left(k_{1}\right)-k_{2} \sqrt{H^{2}+\|\mathbf{q}[n]\|^{2}}\right) \\
\text { s.t } \quad(3)-(7), & \\
& \sum_{\min }^{N} \leq \tau[n], \tau[n]^{2} \leq \phi(\mathbf{v}[n]), \\
& (n=1, \ldots, N),
\end{aligned}
$$

where $\tau$ is a slack variable for solving non-convex part of (2) and $\phi(\mathbf{v}[n])$ is the first Taylor approximation of $\|\mathbf{v}[n]\|^{2}$ at $\mathbf{v}_{j}[n]$ and $\phi(\mathbf{v}[n])=\left\|\mathbf{v}_{j}[n]\right\|^{2}+2 \mathbf{v}_{j}^{T}[n]\left(\mathbf{v}[n]-\mathbf{v}_{j}[n]\right)$. Note that we approximate the numerator of (2) to concave form, using high-SNR property, $k_{1} e^{-k_{2} \sqrt{H^{2}+\|\mathbf{q}[n]\|^{2}}} \gg 1$.

For solving (9), we use the sequential fractional algorithm by iterating (9) with updated $\mathbf{q}_{j}[n]$ and $\mathbf{v}_{j}[n]$.

\section{Numerical Results}

In Figs. 1 and 2, we assumed the altitude $H=100 \mathrm{~m}$, $c_{1}=9.26 \times 10^{-4}, c_{2}=2250$, bandwidth $B=1 \mathrm{MHz}$, initial position $\mathbf{q}_{\mathrm{I}}=[0,1000]^{T}$, end position $\mathbf{q}_{\mathrm{F}}=[1000,0]^{T}$, initial/end velocity $\mathbf{v}_{\mathrm{I}}=\mathbf{v}_{\mathrm{F}}=30\left(\mathbf{q}_{\mathrm{F}}-\mathbf{q}_{\mathrm{I}}\right) /\left\|\mathbf{q}_{\mathrm{F}}-\mathbf{q}_{0}\right\|$, flighttime $T=400$. Figs. 1 presents the comparison of the proposed

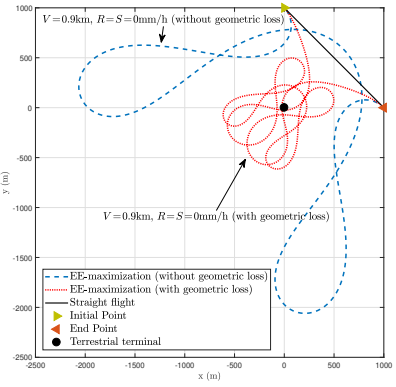

Fig. 1. Energy efficient trajectory considering beam divergence effect.
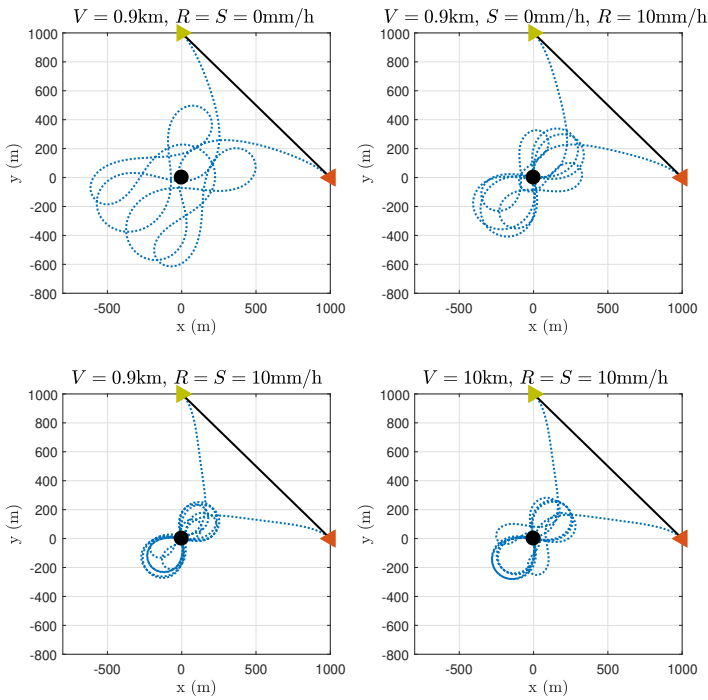

Fig. 2. Energy efficient trajectory considering weather effect.

energy efficient flight paths without and with geometric loss based on beam divergence effect with $D_{R}=8 \mathrm{~cm}, D_{T}=3 \mathrm{~cm}$, and $\theta_{\text {div }}=2 \mathrm{mrad}$. For Fig. 2, each of the four flight paths represents an energy efficient trajectory when the different atmospheric environments are taken into account. We assumed the parameter values in wet snow $a=5.42 \lambda \times 10^{-5}+5.50$, $b=1.38$ [3]. Fig. 1 shows that as the beam divergence effect increases, the channel condition deteriorates and thus the radius of the trajectory becomes narrower. Similarly, Fig. 2 shows that depending on the weather condition, the communication environment changes and the range of energy efficient trajectory changes.

\section{ACKNOWLEDGEMENT}

This work was supported by the National Research Foundation of Korea (NRF) grant funded by the Korea government (MSIT)(NRF-2018R1A2B2007789)

\section{REFERENCES}

[1] Y. Zeng and R. Zhang, "Energy-efficient UAV communication with trajectory optimization," IEEE Trans. Wireless Commun., vol. 16, no. 6, pp. 3747-3760, Jun. 2017.

[2] H. Ghazzai, M. B. Ghorbel, and A. Kadri, "Energy efficient 3D positioning of micro unmanned aerial vehicles for underlay cognitive radio systems," in Proc. International Conf. on Commun., Paris, France, May 2017, pp. 1-6.

[3] H. Kaushal and V. K. J. and S. Kar, Free Space Optical Communication. India: Springer, 1st ed. 2017.

[4] A. Lapidoth, S. M. Moser, and M. A. Wigger, "On the capacity of freespace optical intensity channels," IEEE Trans. Inf. Theory., vol. 55, no. 10 , pp. 4449-4461, Oct. 2009. 